\title{
La Economía Política Internacional en el campo de las Relaciones Internacionales ${ }^{*}$ argentinas
}

\author{
MeLisa DeCIANCIO**
}

Artículo recibido: 3 de noviembre de 2017

Artículo aprobado: 9 de marzo de 2018

Doi: http://dx.doi.org/10.12804/revistas.urosario.edu.co/desafios/a.6106

Para citar este artículo: Deciancio, M. (2018). La economía política internacional en el campo de las relaciones internacionales argentinas. Desafios, 30(2), 15-42. Doi: http://dx.doi. org/10.12804/revistas.urosario.edu.co/desafios/a.6106

\section{Resumen}

Este trabajo busca responder a la pregunta acerca de cuáles han sido los principales aportes de la Economía Política Internacional (EPI) al devenir histórico del campo intelectual de las Relaciones Internacionales (RI) en Argentina. El trabajo se inscribe en dar respuesta a una pregunta más amplia acerca del proceso de constitución del campo de las RI como disciplina académica autónoma en Argentina. Se indagará sobre los aportes realizados por la EPI, especialmente, el estructuralismo latinoamericano y el desarrollismo, como espacios de producción teórica y conceptual desde donde se construye parte del pensamiento sobre la realidad internacional. El objetivo del trabajo

\footnotetext{
* Se hace referencia a las "relaciones internacionales" (con minúscula) al referirse a los vínculos de un país, o miembro de ese país, con otro. "Relaciones Internacionales", en cambio, refiere a la disciplina que estudia esos vínculos.

** Doctora en Ciencias Sociales por la FLACSO, Argentina. Magíster en Relaciones y Negociaciones Internacionales (2010) por la FLACSO/Argentina y la Universidad de San Andrés, licenciada en Ciencia Política (2006) por la Universidad de Buenos Aires. Becaria posdoctoral del CONICET. Actualmente es la coordinadora académica de la Maestría en Relaciones Internacionales de la FLACSO/Argentina y docente en FLACSO, UBA, y USAL. Correo electrónico: mdeciancio@flacso.org.ar. ORCID: http://orcid.org/0000-0001-9616-0671
} 
girará en torno a reconstruir la agenda de EPI y su contribución al desarrollo de las RI argentinas mediante la observación y análisis de los principales autores y conceptos, las instituciones que han servido como espacios de producción de conocimiento y las revistas especializadas que actuaron como plataformas para la difusión de temas e ideas.

Palabras clave: economía política, relaciones internacionales, bistoria, disciplina.

\title{
International Political Economy within the Discipline of International Relations in Argentina
}

\begin{abstract}
This paper seeks to answer the question what have been the main contributions of international political economy (IPE) to the historical evolution of the intellectual field of International Relations (IR) in Argentina. This inquiry is embedded within a broader question about the process of the constitution of the IR field as an autonomous academic discipline in Argentina. In order to do so, I analyze the contributions made by IPE, especially Latin American structuralism and developmentalism, as spaces of theoretical and conceptual production from which part of the thinking about the international reality has been built. The main objective of the paper revolves around reconstructing the IPE agenda and its contribution to the development of Argentine IR through the observation and analysis of the main authors and concepts, the institutions that have served as spaces of knowledge production, and finally the academic journals that have acted as platforms for the dissemination of themes and ideas.
\end{abstract}

Keywords: political economy, international relations, history, discipline.

\section{A economia política internacional no campo das relações internacionais argentinas}

\begin{abstract}
Resumo
Este trabalho busca responder àpergunta acerca de quais têm sido os principais aportes da economia política internacional (EPI) ao devir histórico do campo intelectual das Relações Internacionais na Argentina. O trabalho inscreve-se em dar resposta a uma pergunta mais ampla acerca do processo de constituição do campo das RI como disciplina acadêmica autónoma na Argentina. Se indagará sobre os aportes realizados pela EPI, especialmente o estruturalismo latino-americano e o desenvolvimentismo, como espaços
\end{abstract}


de produção teórica e conceitual desde onde se constrói parte do pensamento sobre a realidade internacional. O objetivo do trabalho estará ao redor de reconstruir a agenda de EPI e a sua contribuição ao desenvolvimento das RI argentinas através da observação e análise dos principais autores e conceitos, as instituições que têm servido como espaços de produção de conbecimento e as revistas especializadas que atuaram como plataformas para a difusão de temas e ideias.

Palavras-chave: economia Politica, Relações Internacionais, história, Disciplina.

\section{Introducción}

Este trabajo propone un recorrido por los aportes de la Economía Política Internacional (EPI) y, en particular, del estructuralismo latinoamericano, a la disciplina de las Relaciones Internacionales (RI). Se inscribe dentro de un trabajo más amplio acerca de los enfoques desde los cuales se ha nutrido el campo de las RI argentinas y la manera cómo se ha ido constituyendo en tanto disciplina autónoma en el país ${ }^{1}$. Se busca retomar las contribuciones realizadas por la economía política a la construcción de una agenda de investigación sobre EPI que marcó uno de los ejes centrales de las RI argentinas: el estudio del regionalismo. Esto, poniendo el foco en los trabajos orientados al análisis del regionalismo latinoamericano de los sesenta y los procesos de integración regional emergentes a partir de las décadas de los ochenta y de los noventa con de la firma del Tratado de Asunción (1994) que dio inicio al Mercosur. En estas páginas se indagará, en primer lugar, sobre el marco histórico de referencia en el cual se inscribe la teoría estructuralista latinoamericana. En segundo lugar, se abordarán, brevemente, el desarrollo de la Economía como campo en Argentina y las características que adquirió la EPI, ambas pilares para los desarrollos posteriores que abonaron el campo de las RI argentinas y latinoamericanas. Tercero, se enfocará la mirada sobre los fundamentos y principales ideas de la EPI en América Latina, sus desarrollos teóricos y el aporte a una perspectiva económica de las RI. Cuarto, se estudiará el papel del Instituto para la Integración de América Latina (INTAL) y de la Revista de Integración como espacios para la producción y difusión del

\footnotetext{
Este trabajo se inscribe dentro de mi tesis doctoral "Historia de la construcción del campo de las Relaciones Internacionales en Argentina” (2016a).
} 
conocimiento sobre EPI en Argentina. Quinto, se esbozarán las líneas de investigación y redes de conocimiento que siguieron a los trabajos sobre regionalismo y que consolidaron la EPI dentro del campo de las RI argentinas. Por último, seguirá una serie de conclusiones.

Diana Tussie (2015) señala que, en Argentina, la EPI tuvo dos estímulos fuertes: el primero, iniciático, marcado por el impulso de la teoría de la dependencia; y, otro más reciente, en los años noventa, con la constitución del Mercosur y de los bloques regionales. Este segundo estímulo dio un tono menos determinista a las investigaciones académicas que a la vez se acercaron a un diálogo y a una interacción más íntima con la política pública. Ambos muestran la gran cantidad de cambios que han marcado el desarrollo de los estudios, otorgándoles sus características propias y alterando su curso (Tussie, 2015)․ Al analizar el caso argentino, resulta desacertado dejar de considerar que gran parte de los desarrollos teóricos que surgieron en este momento trascendieron las fronteras nacionales para convertirse en un fenómeno de escala regional. Es por eso que resulta impreciso abordar estos aportes como exclusivamente nacionales, a pesar de que gran parte del debate estuviera impulsado por Raúl Prebisch, un intelectual argentino. El desarrollo de las RI argentinas, impulsado desde la economía política, se dio por un fenómeno a nivel regional que estimuló los estudios sobre esta y otras ramas de la disciplina en muchos de los países latinoamericanos. Así, en el marco de las contribuciones de la EPI latinoamericana al campo de las RI, las regiones y el regionalismo adquirieron especial relevancia. Esto no implica que esta haya sido la única contribución de la EPI al campo de las RI sino que ha sido la que ha marcado una de las líneas de investigación más relevantes dentro de la disciplina, junto a aquellas más preponderantes como los estudios de política exterior y seguridad internacional. Las versiones latinoamericanas de la sociología del desarrollo y de la economía del desarrollo, fundadas en el estructuralismo, la sociología crítica y la teoría de la dependencia, fueron expresiones de la capacidad de los científicos de la región para confrontar aquellas ideas dominantes en el debate

\footnotetext{
2 Sobre una evolución de la EP en América Latina y Argentina ver Tussie \& Riggirozzi (2015) y Tussie (2015).
} 
internacional cuestionando el saber convencional y transformándolo para reinventarlo (Tussie, 2015).

En términos metodológicos para el análisis, se consideran los agentes de producción de conocimiento (académicos y especialistas) así como los espacios (instituciones, redes, publicaciones) donde se desarrollaron los saberes especializados sobre RI en el país. Se busca rescatar aquellas instituciones, redes, personas y saberes que incidieron en la formulación de nuevas aproximaciones al pensamiento sobre lo internacional. Así, entre los espacios estudiados, se consideran tanto las universidades, en tanto ámbitos de formación y difusión de saberes, como instituciones públicas y privadas de producción del conocimiento y aquellas redes de colaboración que estimularon la proliferación de los estudios internacionales a nivel nacional (Altamirano \& Sarlo, 2001; Buchbinder, 2005; Clark, 1997; Serrano, 1997). Los agentes considerados serán diversos intelectuales vistos como sujetos atravesados en la producción del conocimiento especializado, tanto por el ámbito académico, como por el estatal (Altamirano \& Sarlo, 1997; Plotkin \& Zimmermann, 2012). Por último, se abordan también las revistas especializadas, consideradas mecanismos de difusión y legitimación de saberes (Pita González \& Grillo, 2015; Dosse, 2007). Para ello, se seleccionaron las revistas más representativas dentro del ámbito de la economía política de la época, en Argentina, sin desarrollar exhaustivamente toda la producción, sino aquella que ha tenido mayor impacto y ha servido de fuente de consulta e información ${ }^{3}$.

El siguiente apartado analizará el vínculo entre economía política y el campo de las RI argentinas con el objetivo de vislumbrar los puntos

\footnotetext{
3 Con el fin de cumplir con el objetivo del trabajo, para el análisis de estas revistas se hizo una revisión exhaustiva de cada una de ellas, examinando cada número publicado (y disponible) de cada colección. En esta tarea se observaron los índices completos de las revistas, haciendo un recorte a partir de la selección de aquellos titulares vinculados a temáticas de las relaciones internacionales. A partir de allí, se observaron y sistematizaron las temáticas abordadas y los autores que participan. En consonancia con su interés y relevancia para esta la investigación, se analizaron artículos específicos, los debates en los que se inscribieron y las biografías de los autores.
} 
de contacto entre ambos campos, tanto en términos de su origen, como de problemáticas compartidas.

\section{La Economía Política en el campo de las Relaciones Internacionales}

Desde fines del siglo XIX, las ciencias sociales en general se han visto influenciadas tanto por las condiciones estructurales del país, como por los recursos, la inestabilidad política y las crisis económicas. Desde la crisis de 1873, comenzó a delinearse un cuerpo de especialistas que se consolidó con la de 1890 y con la aparición de instituciones dedicadas a la enseñanza de la Economía. A partir de estos acontecimientos, los economistas pasaron a ubicarse en el centro de la escena estatal cuando tanto el Estado como la sociedad comenzaron a demandar, con más fuerza, saberes expertos que permitieran dar cuenta de lo que estaba sucediendo mientras que, a su vez, estos fueron cobrando cada vez más legitimidad (Plotkin, 2010).

La EPI ha estado siempre vinculada a las RI. En los países anglosajones, donde incluso ambos campos poseen un acervo de gran densidad, la economía política nace en la década de los setenta ${ }^{4}$. A pesar de que en la práctica la economía política siempre estuvo fuertemente vinculada a las RI, hasta ese momento ambas eran tratadas como disciplinas completamente independientes, cada una con su propia perspectiva de los asuntos internacionales. Frieden y Lake (2000) entienden la economía política como el estudio de la interacción entre las arenas política y económica. Mientras la economía puede definirse como el sistema de producción, distribución y uso de la riqueza, la política se considera como el conjunto de instituciones y reglas que gobiernan las interacciones sociales y económicas.

\footnotetext{
4 Entre los más destacados académicos dentro de la Economía Política Internacional anglosajona se encuentran Robert Cox, Robert Gilpin, Peter Katzenstein, Robert Keohane, Charles Kindleberger, Stephen Krasner y Susan Strange. Para una aproximación a sus trabajos y la EP internacional ver Cohen (2008) y Frieden \& Lake (2000).
} 
Mientras en las RI primaba la atención sobre la Guerra Fría, la defensa, y la seguridad, la EPI parte del postulado de que las relaciones internacionales se han desarrollado con indiferencia y hasta negación sobre la gravitación de las fuerzas económicas y de los operadores de mercado. Por ello pone permanentemente en tela de juicio los análisis que presumen una excesiva autonomía de lo político (Tussie, 2015). Para Guzzini, por ejemplo, la EPI surge como una reacción, en parte a favor y en parte en contra, de la mucho más sistémica — pero restringida - teoría neorealista de las RI propuesta por Kenneth Waltz (Guzzini, 1998). Desde Adam Smith, John Stuart Mill y Karl Marx, la economía se considera eminentemente política, a la vez que la política se encuentra atada a los fenómenos económicos. Pero las RI no incorporaron los factores y actores económicos a su centro de atención hasta la caída del sistema de Bretton Woods y la devaluación del dólar, en 1973. La llamada "crisis del petróleo" contribuyó a poner en tela de juicio todos los fundamentos hasta entonces invisibilizados del sistema económico occidental. La preocupación por la declinación económica y sus debates abren paso gradualmente a una mayor confluencia entre las RI y la EPI.

Luego de los setenta, la economía política cobró fuerza a partir de la disconformidad de los académicos con la distancia existente entre los modelos abstractos del comportamiento político y económico y lo que realmente sucedía en la economía y en la política. Paralelamente, las crisis económicas politizaron cada vez más a la economía al tiempo que la preocupación de los sistemas políticos por los factores y actores económicos iba en aumento (Frieden \& Lake, 2000).

$\mathrm{Al}$ igual que sucede con las RI, la EPI se caracteriza por su interdisciplinariedad, con el objeto de tender puentes entre las especialidades más antiguas y establecidas al incorporar perspectivas más frescas sobre el estudio de la economía mundial (Cohen, 2008). Esto hace que ambos campos puedan complementarse mutuamente en un intento por unir las brechas existentes entre ambos.

Del mismo modo, en el caso argentino, la economía y la sociología económica contribuyeron al desarrollo de un enfoque de las RI donde 
se incorporan nuevos actores y procesos a un campo que, como se señaló antes, estuvo tradicionalmente centrado en el Estado como actor y productor de los vínculos con el exterior. De hecho, los profesionales argentinos estuvieron marcadamente internacionalizados en comparación con los de otros países de la región (Dezalay \& Garth, 2010). La incorporación de variables y fuerzas económicas a la dinámica de las relaciones exteriores estuvo principalmente motivada, en sus comienzos, por las propuestas integracionistas regionales al asumirse el lugar periférico del país en las relaciones económicas internacionales. Como resultado, desde los primeros trabajos del ingeniero Alejandro Bunge y su propuesta de creación de una Unión Aduanera del Sud, hasta el proyecto integracionista de la década de los sesenta, encarado por Raúl Prebisch y el desarrollismo latinoamericano, los estudios sobre integración regional han marcado e impulsado a la economía política en Argentina. Así, a mediados del siglo Xx, las tensiones centro-periferia establecieron una nueva comprensión de la política internacional. Al mismo tiempo, el campo de las RI empezaba a ser reconocido como disciplina autónoma de la mano de su institucionalización en las universidades y de un creciente sentido de urgencia con respecto a la política y la dependencia económica de la región (Tickner, 2003).

Hasta entrada la década de los ochenta, la economía política ha marcado el campo de las RI a través de los estudios sobre integración regional y regionalismo, constituyendo también uno de los principales aportes de las RI regionales a la disciplina a nivel global (Acharya, 2011; 2014; Deciancio, 2016b). En cierto modo, para hacer un paralelismo con el proceso europeo, mientras que la teoría de la integración regional europea tiene sus raíces en las ciencias sociales, la teoría de la integración regional latinoamericana tiene sus raíces en la economía política latinoamericana (Perrotta, 2014) y, más concretamente, en una visión regional de la EPI (Tussie \& Riggirozzi, 2015) las cuales se nutren y fundamentan mutuamente.

En el próximo apartado se indagará acerca de las particularidades que adquiere el campo de la economía en Argentina, sus orígenes y la conformación de una línea de trabajo en economía política. 


\section{El campo de la Economía en Argentina y sus principales postulados: el regionalismo en el centro de la agenda internacional}

Desde mediados del siglo XIX, se dio un proceso que puso a los economistas en el centro de la escena estatal con el consiguiente surgimiento de instituciones dedicadas a la enseñanza de la disciplina y de un grupo de profesionales especializados que irían adquiriendo un lugar específico en la vida intelectual argentina (Caravaca, 2010). Tal y como sucediera en otras ramas de las ciencias sociales, la Facultad de Derecho de la Universidad de Buenos Aires tuvo un papel central en la formación de políticos e intelectuales, y la Cátedra de Economía Política se convirtió en el espacio académico por excelencia de difusión de la disciplina (Plotkin, 2010). Comparativamente con otros países de la región como México o Brasil, donde la enseñanza en las universidades comenzó en la década de los treinta, la Universidad de Buenos Aires fue pionera al crear la carrera de Ciencias Económicas en 1913.

A pesar de que la especialidad de economía política no estuvo definida como disciplina autónoma a nivel universitario por un largo periodo, ya desde tiempos tempranos se pueden detectar debates dentro de la facultad acerca de las características de la Economía como disciplina (particularmente centrados alrededor del lugar asignado a las matemáticas), sus vínculos con el diseño de políticas y, más en general, sobre la naturaleza de dichas políticas. La Revista de Ciencias Económicas (1958-1963) contribuyó al debate, al que luego se sumaría la Revista de Economía Argentina (1918-1952), publicada por el profesor de la facultad, el ingeniero Alejandro Bunge (Plotkin, 2006).

Bunge adquirió especial relevancia para el campo de las RI y la construcción de una agenda de investigación sobre regionalismo a partir de su propuesta de creación de una Unión Aduanera del Sud. De hecho, la EPI ha realizado sus principales aportes al campo de las RI, en su etapa de constitución, en torno a la integración regional y al regionalismo. En este marco, el desarrollo más relevante provino de las ideas y trabajos de uno de los jóvenes colaboradores de Bunge en la Facultad de Ciencias Económicas, Raúl Prebisch. Gerente del Banco Central de Argentina y posterior secretario general de la recién 
creada Comisión Económica para América Latina y el Caribe (CEPAL), Prebisch presentó el primer desarrollo teórico autóctono marcado por el estructuralismo económico y la escuela latinoamericana de la dependencia. Se centró en los grandes proyectos de desarrollo económico a escala nacional sobre la base de políticas de industrialización y sustitución de importaciones a través de visibilizar las diferencias estructurales entre centro y periferia, y propulsó la integración regional como mecanismo para la inserción internacional de los países latinoamericanos. Esta visibilización de las tensiones centro-periferia establece una nueva comprensión de la política internacional en la mitad del siglo xx. Bajo la insignia del desarrollo se desenvolvió el que podría ser el intento más original de abordar colectivamente —desde perspectivas propias - el diagnóstico y las propuestas de futuros posibles para las sociedades latinoamericanas. Bajo la insignia del desarrollo y los conceptos de centro-periferia se sentaron las bases de una sociología económica y una economía política en la región (Tussie, 2015).

Bajo este impulso, la idea de América Latina como un todo comenzó a cobrar cada vez más fuerza y sentó las bases de un pensamiento regional apoyado en el desarrollo económico del continente. Estas nociones surgieron como una reacción en contra de la teoría producida por Estados Unidos acerca de las etapas de desarrollo y de la teoría de la modernización (Lipset, 1959; Gunder Frank, 1970; O’Donnell, 1973) 5 . De la mano de las teorías estructuralistas y dependentistas surgió una serie de análisis orientados a observar el lugar que América Latina debía ocupar en el sistema internacional, tanto en términos de alianzas políticas como del desarrollo económico regional y de la inserción internacional. En esta línea, cobró especial relevancia la idea de integración regional, que trajo consigo la de que la expansión industrial endógena podría surgir de los polos de crecimiento designados estratégicamente

\footnotetext{
5 La teoría de la modernización cobró fuerza entre las décadas de los cincuenta y de los sesenta en las ciencias sociales de Estados Unidos como abordaje a los problemas de desarrollo. Busca explicar el subdesarrollo y la modernización como procesos lineales a través de los cuales las sociedades incorporan los valores de Occidente que les permiten hacer la transición de las sociedades tradicionales a las modernas. Sobre teoría de la modernización ver Rostow (1961); y para su abordaje desde Argentina, ver Di Tella \& Zymelman (1967).
} 
y ubicados con respecto a los centros urbanos y las redes logísticas (Scott, 2009). Sin embargo, el regionalismo se ha entendido durante este período en términos de instrumentos para mejorar el rendimiento económico y la conciencia del hecho de que la formación de bloques económicos en otras regiones del mundo podría poner en peligro el futuro de los países latinoamericanos (Mace, 1988; Briceño-Ruiz, 2007). Así, el regionalismo surgió como uno de los principales legados de la economía política a las RI argentinas y latinoamericanas, como uno de los conceptos que más se estudiarían en períodos posteriores de la disciplina, y como uno de los principales aportes de las RI regionales a la disciplina a nivel global (Acharya, 2011; 2014). Durante el proyecto estructuralista, el regionalismo estuvo marcado por principios económicos y por la búsqueda de una inserción menos asimétrica en la economía global y sus instituciones de posguerra.

Las regiones y el regionalismo han sido centrales en las RI latinoamericanas. Varios estudiosos latinoamericanos se acercaron a la teoría de las RI a través del lente de regionalismo y de la EPI, y se abocaron a la construcción de una agenda de investigación más adecuada para explicar las realidades de la región, rechazando el enfoque liderado por los procesos de integración europea (Börzel \& Risse, 2009). Los debates teóricos de las RI latinoamericanas se han construido en gran medida sobre los diversos enfoques del regionalismo, centrados en el objetivo de obtener una mejor posición en los asuntos mundiales manteniendo la autonomía del subcontinente.

A continuación se explorarán los principales abordajes teóricos de la economía política en América Latina y su contribución a la construcción de una línea de investigación sobre EPI dentro del campo de las RI argentinas.

\section{La Economía Política latinoamericana}

Uno de los aportes más significativos de la EPI a la construcción del campo de las RI argentinas proviene de los debates sobre el desarrollo surgidos en las décadas de los cincuenta y sesenta. Los debates teóricos de las RI latinoamericanas se han construido en gran medida sobre 
los diversos enfoques del regionalismo, centrados en el objetivo de obtener una mejor posición en los asuntos mundiales y manteniendo su autonomía (Deciancio, 2016b). Con el proyecto estructuralista, el regionalismo estuvo marcado por principios económicos y por la búsqueda de una inserción en la economía global y sus instituciones de posguerra.

\section{Teoría de la dependencia}

Fernando Henrique Cardoso y Enzo Faletto sentaron las bases de la teoría de la dependencia a partir de la publicación de su obra Dependencia y desarrollo en América Latina con el objetivo de destacar la naturaleza social y política de los problemas del desarrollo en la región. Se trata, tal vez, del principal y más importante esquema teórico elaborado desde la periferia frente a los problemas de desarrollo e inserción internacional. A pesar de no haber sido pensada en sus orígenes como una teoría de las relaciones internacionales, sus postulados ofrecen una perspectiva latinoamericana para el análisis de las relaciones políticas y, principalmente, económicas de las relaciones transfronterizas - sobre todo por su foco en las relaciones entre Estados-, el desarrollo económico nacional y la soberanía (Tickner, 2003).

Los teóricos de la dependencia veían los procesos de desarrollo estatal íntimamente relacionados con la lógica del sistema capitalista (Sunkel, 1980). Según ellos, una de las principales manifestaciones de la dependencia se revela al nivel del Estado, cuya consolidación se veía limitada por la naturaleza cambiante del capitalismo. El desarrollo nacional era visto como el principal objetivo de los países periféricos y la división internacional del trabajo constituye el principal obstáculo para alcanzarlo. Así lo señalaban Cardozo y Faletto (1977):

[...] la situación de subdesarrollo se produjo históricamente cuando la expansión del capitalismo comercial y luego del capitalismo industrial vinculó a un mismo mercado economías que, además de presentar grados diversos de diferenciación del sistema productivo, pasaron a ocupar posiciones distintas en 
la estructura global del sistema capitalista. De ahí que entre las economías desarrolladas y las subdesarrolladas no solo exista una simple diferencia de etapa o de estado del sistema productivo, sino también de función o posición dentro de una misma estructura económica internacional de producción y distribución (Cardoso \& Faletto, 1977, p. 12).

Los propulsores de la teoría han prestado especial atención al papel de la historia como determinante de las posibilidades y de las barreras del desarrollo: era necesario, sostenían, observar la manera en que las economías subdesarrolladas se vincularon históricamente al mercado mundial y en que se constituyeron los grupos sociales (las clases dominantes) que permitieron preservar las relaciones de dependencia con el centro. La dependencia no era para ellos un estado inalterable, sino que se encontraba en constante cambio como resultado de las transformaciones en los modos de producción característicos de cada país.

De acuerdo con David L. Blaney (1996, p. 461), la teoría de la dependencia pensó a la sociedad internacional como la combinación de dos lógicas: la lógica del capitalismo y la lógica de la soberanía, siendo esta última una precondición para el desarrollo (Blaney, 1996, p. 466). Aunque la mayoría de los autores agrupados bajo el paraguas de la dependencia fallaron al hacer referencia explícita al problema de la soberanía (Tickner, 2003), su tratamiento del papel del capitalismo y del imperialismo en el establecimiento de las reglas del juego económicas y políticas en la periferia permitió llegar a la conclusión de que una de las características definitorias de la dependencia es precisamente la ausencia de soberanía (Cardoso \& Faletto, 1977).

Teniendo en cuenta estas importantes contribuciones en un nivel más práctico, la teoría de la dependencia no logró producir una solución viable a las situaciones de dependencia, a menos que se hiciera la revolución, se adoptara una forma de producción socialista o se aceptara un desarrollo dependiente y desviado. Cardoso y Faletto (1977, p. 29) dejaron esto claro al sostener que en el proceso de tira y afloje entre las fuerzas sociales y el mercado, característico de la construcción de 
Estados, las fuerzas del mercado internacional siempre limitan las posibilidades de toma de decisión y acción autónoma.

La teoría de la dependencia abonó gran parte del pensamiento latinoamericano desde la década de los sesenta. La construcción de una visión de Ri pensada desde "los débiles" surgió como parte de un enfoque que se dio a nivel regional en una búsqueda por desarrollar un pensamiento propio y reducir la dependencia económica, política e intelectual. Desde esta perspectiva, contribuyó a que en Estados Unidos y en otros lugares del mundo comprendieran las relaciones en el sistema internacional, y la relevancia de las asimetrías y sus efectos. Como resultado, la teoría de la dependencia no solo fundamentó gran parte de los trabajos económicos de la época sino que, aunque produjo pocos proyectos de investigación empíricos en relaciones internacionales (Lowenthal, 1983), sirvió de base conceptual para el desarrollo de una de las principales teorías de política exterior esbozadas desde Argentina: la autonomía heterodoxa de Juan Carlos Puig.

\section{La EPI latinoamericana y las RI desde la periferia. La escuela estructuralista-desarrollista}

Una cuestión que ocupó un lugar preponderante en la política de Argentina hacia América Latina durante principios de la década de los sesenta fue el acercamiento a Brasil y la búsqueda de una estrategia de cooperación tanto política como económica sin precedentes con dicho país. Con la firma de los acuerdos de Uruguayana, el 22 de abril de 1961, ambos países se comprometieron a "mantener un intercambio de informaciones de carácter relevante en el ámbito internacional" (Art. 2, Acuerdo de Uruguayana) y se concluyó que debían aumentar su cooperación comercial en paralelo con el acelerado proceso de industrialización. Su principal objetivo era concertar una acción internacional conjunta "en función de la condición sudamericana que les es común" (Acuerdo de Uruguayana). Con la firma del Tratado de Montevideo, en 1960, se dio origen a la ALALC - enmarcada en las ideas que, por aquel entonces propiciaban la CEPAL y su mentor, Raúl Prebisch—, y se impulsó la creación de la primera experiencia de integración latinoamericana de la posguerra. 
Frondizi y el desarrollismo argentino no apoyaron la creación de un mercado común latinoamericano, como proponía la CEPAL, a pesar de que para ese momento ya el $16 \%$ de las exportaciones argentinas tenía ese destino. De este modo surgió la ALALC como un área de librecambio con desgravaciones reciprocas, graduales y progresivas que buscaban liberalizar sustancialmente todo el comercio latinoamericano en un periodo de doce años.

En simultáneo, con la presidencia de Arturo Illia (1963-1966), a nivel global comenzó a ganar terreno el debate Norte-Sur por la reforma del sistema de comercio internacional. En 1962, había surgido el Grupo de los 77 (países en desarrollo) que se había congregado en Naciones Unidas para motorizar la reforma, y que se apuntalaba en la coordinación regional articulando la periferia. Así, esta surgió por primera vez como actor político-económico a nivel mundial; empieza a congregarse en foros internacionales en la defensa de sus intereses comunes. Se formaron, entonces, el grupo africano, el asiático y, en América Latina, el llamado GrulA. Con la llegada de Illia al gobierno, en 1963, Argentina hizo un esfuerzo por liderar el GRULA, aprovechando los lazos con la CEPAL que, en ese momento, vivía su edad de oro. En su afán por lograr protagonismo a nivel regional, en 1964, tuvo lugar, en la localidad cordobesa de Alta Gracia, la reunión convocada por la Comisión Especial de Coordinación Latinoamericana (CECLA), instancia previa al lanzamiento de la Conferencia de Naciones Unidas sobre Comercio y Desarrollo (UNCTAD I). Esta reunión fue presidida por el ministro de Economía de Illia, Eugenio Blanco (cercano a Prebisch) y contó con la asesoría de figuras vinculadas a la Comisión Económica para América Latina y el Caribe (CEPAL) y al enfoque desarrollista latinoamericano, como Alfredo Concepción, Bernardo Grinspun o Leopoldo Tettamanti, entre otros. Allí se aprobó la llamada Carta de Alta Gracia, documento inspirado en las ideas de Raúl Prebisch, en la cual 19 países latinoamericanos denunciaron el carácter discriminatorio de la estructura del comercio internacional como el factor responsable de la vulnerabilidad de los países en vías de desarrollo. Con ello, la polarización Norte-Sur comenzaba a prevalecer y dejaba a la disputa Oriente-Occidente en un segundo plano. 
El economista Raúl Prebisch y la CEPAL se abocaron a la tarea de crear una conciencia colectiva de los problemas económicos que afrontaba la región mediante un análisis de los mecanismos que los perpetuaban y se convirtieron en voceros de esa nueva conciencia en los foros mundiales. El desarrollismo, como se llamó a esta corriente, basó su propuesta en el ensanchamiento del sector industrial que produce bienes de consumo duraderos, en particular de la industria del automóvil, mediante la instalación de ramas locales de empresas productoras norteamericanas y europeas.

La economía política latinoamericana estuvo impulsada y marcada por las ideas económicas promulgadas por dos organizaciones internacionales cuya área de alcance y membresía comprendía a los países de América Latina: la CEPAL y ALALC. La CEPAL surgió en 1948 como un poderoso think-tank y como foro para el diálogo y la negociación, una plataforma para la cooperación y la cohesión y una fuente de información y asesoramiento a los gobiernos de América Latina (Dembicz, 2004). "Bajo el liderazgo de Prebisch y una coalición de tecnócratas y políticos reformistas, la comisión fue la defensora más fuerte de la integración económica en la región durante toda la década de 1950 y la mayor parte de la década de 1960" (Mace, 1988, p. 408), con la convicción de que los derechos económicos y la cooperación eran los únicos medios para reducir la dependencia tradicional sobre el comercio de exportación de productos primarios (Malamud, 2010). En su "manifiesto" —como lo llamó Albert Hirschman (1968)— "Desarrollo de América Latina y sus principales problemas", Prebisch (1950) contribuyó con un análisis original del sistema internacional que marcaría la economía política latinoamericana e internacional y que estableció las bases de las escuelas de pensamiento estructuralista y desarrollista en la región. Mientras la EPI de Estados Unidos se apoyó en la tradición liberal, las teorías económicas clásicas y sus concepciones subyacentes de simetría, en América Latina, tanto los intereses como los abordajes se apoyaron en las teorías marxistas del imperialismo, en el análisis de la dependencia estructural, y en los hechos y perspectivas de asimetría (Lowenthal, 1983). 
Animados por la CEPAL, políticos y académicos de América Latina comenzaron a concebir la integración regional como una forma de superar las debilidades estructurales y la limitación de los pequeños mercados nacionales. La idea era ampliar la planificación industrial a un nivel de escala en toda la región para eliminar las barreras al comercio recíproco, manteniendo altos niveles de protección externa que sirvieran como un incentivo a la industrialización, al crecimiento económico y a la inversión (Tussie, 2009). En su papel, la CEPAL llevó a la búsqueda de una nueva conceptualización de la "región" y al desarrollo regional basado en la ampliación de los mercados nacionales mediante la constitución de un mercado común y con "el objetivo de sostener el esfuerzo de integración en América Latina para los próximos veinte años" (Mace, 1988, p. 408). Prebisch trató de explicar el subdesarrollo de la región a través del análisis de los efectos del sistema de intercambio capitalista internacional. Debido a términos de intercambio desfavorables, el mero crecimiento impulsado por las exportaciones ya no era viable y por eso este autor abogó por el desarrollo y la industrialización hacia adentro como una manera de reducir la vulnerabilidad de las economías de América Latina. Además, Prebisch afirmó que la unificación de los mercados podría generar ganancias de productividad y acelerar la industrialización, con la condición de que estuvieran protegidos por aranceles elevados. La recomendación fue lanzar una estrategia de industrialización por sustitución de importaciones (ISI) colectiva a nivel regional (Dabène, 2012).

Como sintetiza Mace (1988), la tesis de la CEPAL proponía examinar la economía mundial en una estructura compuesta por un centro y una periferia esencialmente unidos por relaciones comerciales que se caracterizaban por el deterioro de los términos de intercambio. Este estado de cosas fue el factor responsable del desarrollo desigual en la economía mundial y en particular de la dependencia de América Latina. Desde esta perspectiva, para liberarse de esta limitación, la región tenía que industrializarse y la mejor manera de hacerlo era adoptando una política de sustitución de importaciones. Pero esta tuvo resultados insuficientes a nivel nacional debido al limitado alcance de la mayoría de los mercados nacionales. Por lo tanto, al ofrecer mercados regionales más grandes, la integración era el dispositivo más útil para 
lograr la sustitución de importaciones y, en última instancia, la industrialización en el conjunto de América Latina (Mace, 1988, p. 408).

La región fue delineada en estos abordajes como una unidad económica, como la periferia, como sujeto de subdesarrollo. Todos sus países enfrentaban los mismos desafíos que surgían de las relaciones asimétricas entre el centro y la periferia como resultado de la expansión del capitalismo, la división internacional del trabajo y la inserción de las economías latinoamericanas en el sistema global como proveedor de productos que sufrían de deterioro de los términos de intercambio. Según Prebisch:

No es de extrañar entonces que prevalezca frecuentemente en los estudios que suelen publicarse acerca de la economía de los países de América Latina, el criterio o la experiencia especial de los grandes centros de la economía mundial. Mal cabría esperar de ellos soluciones que nos conciernen directamente. Es pertinente, pues, presentar con claridad el caso de los países latinoamericanos, a fin de que sus intereses, aspiraciones y posibilidades, salvadas desde luego las diferencias y modalidades específicas, se integren adecuadamente en fórmulas generales de cooperación económica internacional (Prebisch, 1950, p. 2).

Este enfoque desarrollista y estructuralista de la economía política inspiró varias iniciativas en toda la región así como en otras subregiones. El primer proyecto comercial de este tipo fue ferozmente impugnado por Estados Unidos, pero finalmente logró materializarse en 1960 con la creación de la ALALC, establecida por la ratificación del Tratado de Montevideo, en 1960. El objetivo principal de este bloque fue la creación de una zona de libre comercio en un período de doce años. El Mercado Común Centroamericano y el Banco Interamericano de Desarrollo también vieron la luz en este momento con el mismo espíritu. Para todos, la Comunidad Económica Europea fue una fuente de inspiración. La década de los sesenta se cerró con el intento de convocar a Argentina, Brasil, Uruguay y Paraguay para "aunar sus esfuerzos para el desarrollo armónico y la integración física de la cuenca del Río de la Plata”, lo que refleja las preocupaciones 
estratégicas de los regímenes militares (Dabène, 2012). Sin embargo, los proyectos de comercio se estancaron desde el principio.

El regionalismo de la CEPAL ha sido conceptualizado por gran parte de la bibliografía clásica como "regionalismo cerrado". Sin embargo, este argumento ha sido rechazado por dicha escuela, ya que apuntaba a intensificar el comercio interamericano sin perjuicio de la expansión del comercio en otras áreas con el fin de aumentar el comercio mundial en general. Aunque al principio el plan era proteger la producción regional para aumentar la competitividad a través del comercio regional y la competencia entre iguales, se esperaba que la apertura a los titanes en los mercados mundiales tuviera lugar en las fases posteriores (Dosman, 2008). Briceño-Ruiz llamó a esta fase del regionalismo latinoamericano "regionalismo autonómico". Es decir, en un contexto político, marcado por la aparición de una nueva corriente tecnocrática de América Latina, el objetivo era incrementar la autonomía regional en relación con los centros de poder mundial (Briceño-Ruiz, 2007). El regionalismo latinoamericano, ahora expresado en lenguaje económico a través de los pensamientos seminales y la acción vigorosa de Raúl Prebisch y de la CEPAL, surgió una vez más como una gran resistencia a la intervención extranjera y como búsqueda de la autonomía.

Las principales preocupaciones de Prebisch eran el desarrollo económico, la situación latinoamericana en el sistema internacional y el subdesarrollo regional, temas que habían sido históricamente excluidos de los debates de RI estrictamente centrados en las cuestiones geopolíticas y de defensa - material y legal — del territorio y la autonomía. De hecho, en los debates de RI, la EPI ha sido especialmente excluida de la agenda central, desplazada por un enfoque realista clásico centrado en el Estado como principal actor que separaba las esferas política y económica en el estudio de los asuntos internacionales (Morgenthau, 1948). Además, la disciplina se concentra en las grandes potencias que sientan las reglas relegando al resto del mundo al papel de pasivos receptores. Por el contrario, el enfoque teórico de Prebisch pone la atención en la reforma de reglas y, por lo tanto, en la acción de quienes las reciben. Ubicar el "desarrollo económico" 
a la vanguardia de las negociaciones internacionales ha sido un pilar fundamental en las RI y de la EPI de América Latina, ya que se considera el desarrollo (o la falta de él) el reflejo de las condiciones en la estructura internacional. Los países del centro habían organizado todo el sistema con el fin de satisfacer sus propios intereses, mientras que la periferia se mantuvo pasiva, conectada con el centro sobre la base de la demanda de sus recursos naturales (Prebisch, 1950).

El desarrollo de una EPI en Argentina estuvo impulsado y acompañado por una serie de instituciones y mecanismos de difusión de ideas como la Revista de Integración (1975-1995) que sirvieron de plataformas para fortalecer esta línea de investigación dentro del campo de las RI argentinas. La próxima sección indagará sobre estos espacios.

\section{Espacios de generación y difusión de ideas: el regionalismo como impulso de la EPI dentro del campo de las RI}

\section{El Instituto para la Integración de América Latina (INTAL) y la revista Integración Latinoamericana (1975-1995)}

La escuela de la dependencia, así como el desarrollo teórico de Prebisch y sus aportes al regionalismo permearon el campo de las RI con enfoque economicista. La creación del Banco Interamericano de Desarrollo (1961) y el entusiasmo latinoamericano por los proyectos de integración regional — que se habían materializado en la creación de la ALALC en 1960 - condujeron a la creación del Instituto para la Integración de América Latina (INTAL), en 1963, con sede en Buenos Aires, desde donde se condujeron numerosas investigaciones y estudios ${ }^{6}$. El instituto nació como reflejo de las instituciones europeas especializadas en integración regional y ante la necesidad de dilucidar los problemas que surgieran de la integración así como para crear los cuadros a nivel nacional y regional que hicieran posible la realización del proceso. De sesgo marcadamente desarrollista, el INTAL se propuso la tarea de llevar adelante investigaciones, enseñanza, asesoría y difusión sobre

${ }_{6}$ Ver: http://www.iadb.org/es/intal/creacion-del-intal,7130.html 
integración latinoamericana para contribuir a la formación de funcionarios públicos y privados vinculados al proceso de integración, así de como especialistas para las universidades y otros centros de enseñanza. Desde su creación, el INTAL realizó seminarios de líderes latinoamericanos en el campo económico, social y político, destinados al análisis de los problemas relacionados con la integración de América Latina, así como de la difusión de documentos y estudios sobre estos temas. Como resultado, se abrió un nuevo campo para la investigación que nutrió los estudios latinoamericanos e internacionales en la región. El INTAL se perfilaba en tanto conciencia y escuela del proceso de integración latinoamericana y como un centro motor de la investigación y la docencia en este campo.

En el marco de esas tareas, lanzó una de las revistas más destacadas sobre integración en América Latina, la revista Integración Latinoamericana (19751995). Esta publicación cubrió todo el espectro de temas considerados relevantes sobre la integración regional. Su comité editorial estaba integrado por Natalio Botana, Fernando Mateo, Eduardo White y Luciano Tomassini, uno de los más destacados especialistas en RI en la región. Cada número incluía un editorial y luego diversas secciones: "Estudios" (donde se presentaban los trabajos académicos de especialistas en integración regional, proveyendo de herramientas teóricas y metodológicas), "Notas y comentarios", "Información latinoamericana" (con la actualización de datos sobre los procesos de integración latinoamericanos en curso), "Información internacional", "Documentación y estadísticas", "Actividades del INTAL", "Historia latinoamericana" y "Bibliografía".

En los 207 números que se publicaron en sus casi veinte años de existencia ${ }^{7}$, contó con la colaboración de especialistas, latinoamericanos y anglosajones, en integración regional, comercio internacional, política exterior y economía política internacional. Entre ellos, se encontraban Gustavo Magariños, Felipe Herrera, Carlos Díaz Alejandro, Luciano Tomassini, Gustavo Lagos, Raúl Prebisch, Phillip Schmitter, Félix Peña, Ricardo French Davis, Juan Antonio Valeiras, Enrique Iglesias,

A partir de 1995, con la salida del número especial 207, la revista dejó de publicarse. En el año 1996 fue reemplazada por la nueva revista Integración y Comercio que aún se publica. 
Antonio Ortíz Mena, Carlos Correa, Diana Tussie, Helio Jaguaribe, Daniel Chudnosvky, Samuel Licheszstein, Alieto Guadagni, Manuel Agosin y Natalio Botana entre muchos otros ${ }^{8}$. La revista Integración Latinoamericana fue la base sobre la que se construyeron los estudios sobre integración regional en América Latina y sentó también los cimentos para los estudios sobre comercio internacional que habían comenzado a cobrar fuerza a partir de la firma del Acuerdo General de Aranceles y Comercio en 1947 y, en la región, a medida que los países se fueron incorporando a él.

\section{La herencia de la EP latinoamericana: el RIAL y el CEDES}

Sin duda, el desarrollo teórico planteado por Prebisch marcó una manera de abordar las relaciones económicas internacionales desde la periferia. El crecimiento del área de la integración regional abrió camino a los estudios sobre comercio internacional y, más tarde, a la EPI. Diana Tussie — una de las personas que más ha trabajado sobre EPI en la región— señala que, en nuestro país, esta disciplina tuvo dos impulsos fuertes: el primero, iniciático, marcado por el estímulo de la teoría de la dependencia; y otro más reciente, en los años noventa, con la constitución del Mercosur y los bloques regionales. Este último dio un tono menos determinista a las investigaciones académicas que a la vez se acercaron a un diálogo y a una interacción más íntima con la política pública. Ambos muestran la gran cantidad de cambios que han marcado el desarrollo de los estudios, otorgándoles sus características propias y alterando su curso (Tussie, 2015) ${ }^{9}$. Este enfoque, a menudo olvidado por el mainstream de la disciplina en el país (Tickner, 2003; Tussie, 2004, 2015), sentó las bases de grandes líneas de investigación dentro del campo de las RI.

A los dos impulsos detallados anteriormente se agrega uno intermedio, alejado de las perspectivas sobre integración comercial, que

\footnotetext{
8 Para una revisión completa de títulos y autores ver Integración Latinoamericana, 1995, No. 207, BID-INTAL.

9 Sobre una evolución de la EP en América Latina y Argentina ver Tussie \& Riggirozzi, 2015; Tussie, 2015.
} 
surgió hacia mediados de los setenta y principios de los ochenta, cuando la EPI fue una de las áreas de investigación que más se desarrolló dentro del campo de las RI (Lowenthal, 1983). En línea con el pensamiento desarrollado con anterioridad, comenzaron a realizarse significativos trabajos por parte de economistas y sociólogos sobre el lugar que debía ocupar América Latina en la economía mundial. A los trabajos ya existentes se sumaron, marcados por la nueva coyuntura de endeudamiento de principios de los ochenta, los análisis sobre finanzas internacionales. Comenzó a realizarse una serie de estudios sobre temas como la cambiante división internacional del trabajo y las ventajas comparativas variables, el papel y el efecto del sistema financiero internacional y su vínculo con determinados países latinoamericanos y sus decisiones políticas, los mercados de commodities y regímenes, la EP latinoamericana de industrias específicas, y todos los temas comprendidos por las relaciones mutantes entre las economías dinámicas, abiertas y semiindustrializadas de América del Sur y las lentas, proteccionistas e industrializadas economías de la OCDE (Lowenthal, 1983).

Gran parte de los trabajos mejor desarrollados sobre EPI en los setenta y ochenta en Argentina provinieron de los economistas que estudiaban la economía global en lugares como el Centro de Estudios de Estado y Sociedad (CEDES). Fundado en 1975, el CEDES albergó a un grupo significativo de investigadores que continuaron realizando sus tareas incluso durante la dictadura militar. Estos investigadores comenzaron a centrarse en cuestiones relacionadas con las consecuencias de la apertura e inserción económica internacional de América Latina a costa del aumento de la dependencia y de la mala distribución. Así, observaron el papel del capital extranjero, las alianzas nacional-internacionales en estas nuevas aperturas y la relación entre estas y los regímenes autoritarios, el impacto doméstico de los programas antiinflacionarios impuestos desde el exterior y la naturaleza del período de transición bajo modelos de crecimiento desequilibrado. Con el aumento de la apertura de las economías latinoamericanas a las tendencias inflacionarias y recesiones globales, los académicos latinoamericanos se interesaron cada vez más en la restructuración de los mercados internacionales y comenzaron a buscar modelos macroeconómicos alternativos para interpretar los 
ciclos económicos internacionales en términos de su impacto específico sobre las estrategias del desarrollo en los países semiindustrializados (Lowenthal, 1983).

Paralelamente a las tareas desarrolladas desde el CEDES, con un esfuerzo manifiesto por acercarse a los postulados teóricos y metodológicas de las RI, la EPI fue incorporada en el Programa de Estudios Conjuntos sobre las Relaciones Internacionales de América Latina (RIAL), en 1977. Dicha movida estratégica dio otro sello de origen a la EPI en América Latina, con fuerte sesgo hacia la búsqueda de resquicios para la construcción de políticas públicas. Sus análisis se concentraban en las relaciones de poder asimétricas y en un esfuerzo por operacionalizar los mecanismos que cristalizan las asimetrías, así como las políticas para el cambio. La mirada se volcó de lleno hacia el gigante americano y así surgió un campo de estudio muy dinámico en su momento, focalizado en la economía de Estados Unidos y en su proyección sobre la región. Se postulaba que la única vía posible para romper con las relaciones de subordinación era generando recursos e imaginación política para un nuevo estilo de inserción (Tussie, 2015; Tussie \& Riggirozzi, 2015).

\section{Consideraciones finales}

Los enfoques sobre economía política que surgieron de la mano de la teoría estructuralista latinoamericana han tenido un gran impacto en el campo de las RI. No solo incorporaron una nueva manera de aproximación a los fenómenos internacionales, sino que su visión sobre la integración latinoamericana y los procesos de coordinación regional inauguraron una nueva línea de investigación que cobró gran fuerza en la disciplina y muy particularmente en Argentina. La teorización desde el Sur ha tendido a estar construida de manera preventiva, ya sea como una forma de resistencia o como una manera de encajar en un patrón de "normalidad". En este sentido, la experiencia latinoamericana tiene mucho que añadir a los enfoques regionales de las RI tanto a nivel nacional como regional y global. 
La economía política latinoamericana inaugurada por Prebisch imprimió una perspectiva autóctona a los enfoques sobre lo internacional, al pensar y pensar-se desde la periferia en relación con el mundo. En este sentido, se retomó en parte la tradición jurídica heredada (Deciancio, 2016c), según la cual los países de América Latina necesitan pensar en sí mismos como una entidad única con el fin de garantizar su autonomía y contrarrestar el legado de intervenciones extranjeras. Este factor común ha afectado e influenciado la forma en que la región se relaciona con el mundo y el desarrollo de una estrategia defensiva contra la injerencia extranjera, manifiesta en la formulación de un conjunto sólido de marcos teóricos sobre dependencia y autonomía. El desarrollo de un enfoque de centro-periferia introdujo una idea acerca de cómo los países de la región en desarrollo podrían llevar a cabo una estrategia para sacar el máximo provecho de su posición asimétrica en la distribución internacional de la riqueza y del poder en la gobernanza económica mundial.

La consolidación misma del campo de las RI en América Latina respondió así cada vez menos a un futuro deseado que a la necesidad de reducir los niveles de dependencia política, económica e intelectual de la región, para crear un pensamiento autónomo. Con este énfasis menos normativo y juridicista, el pensamiento internacional latinoamericano buscó superar el determinismo de la dependencia o de las visiones marxistas y las limitaciones en la aplicabilidad de las teorías tradicionales de RI para pensar la política internacional desde el punto de vista de los débiles que no necesariamente marcan la agenda global pero deben comprenderla y tienen de todas maneras grandes campos de acción.

\section{Referencias}

Acharya, A. (2011). Dialogue and discovery: In search of International Relations theories beyond the West. Millennium: Journal of International Studies, 39(3), 619-637.

Acharya, A. (2014). Global International Relations (IR) and regional worlds. A new agenda for international studies. International Studies Quarterly, $58,647-659$. 
Altamirano, C. \& Sarlo, B. (1997). La Argentina del Centenario: campo intelectual, vida literaria y temas ideológicos. En: Ensayos Argentinos. De Sarmiento a la Vanguardia (pp. 102-120). Buenos Aires: Ariel.

Blaney, D. (1996). Reconceptualizing autonomy: The difference dependency theory makes. Review of International Political Economy, 3(3), 459-497. Recuperado de http://www.jstor.org/stable/4177196

Börzel, T. \& Risse, T. (2009). Diffusing (inter-) regionalism. The EU as a model of regional integration. KFG Working Paper Series, septiembre, 7.

Briceño-Ruiz, J. (2007). La integración regional en América Latina y el Caribe. Procesos históricos y realidades comparadas. Caracas: Centro Editorial Litorama CA.

Buchbinder, P. (2005). Historia de las universidades argentinas (2a Ed.). Buenos Aires: Sudamericana.

Caravaca, J. (2010). Algunas claves para pensar el proceso de formación del campo de la economía estatal en la Argentina. Boletín Bibliográfico Electrónico del Programa Buenos Aires de Historia Política, 3(6), 14-15.

Cardoso, F. H. \& Faletto, E. (1977). Desarrollo y dependencia en América Latina. Buenos Aires: Siglo XXI Editores.

Clark, B. (1997). Las universidades modernas. Espacios de investigación y docencia. México: UNAM.

Cohen, B. (2008). International political economy. An intellectual history. Princeton/Oxford: Princeton University Press.

Dabène, O. (2012). Explaining Latin America's fourth wave of regionalism. Regional integration of a third kind. Ponencia presentada en el congreso de la Congress of the Latin American Studies Association (LASA) de 2012. San Francisco, 25 de mayo de 2012.

Deciancio, M. (2016a). Historia de la construcción del campo de las Relaciones Internacionales en la Argentina. Tesis doctoral presentada ante la FLACSO, $1^{\circ}$ de marzo de 2016.

Deciancio, M. (2016b). International Relations from the South: A regional research agenda for global IR. International Studies Review, 1-13.

Deciancio, M. (2016c). Las Facultad de Derecho y Ciencias Sociales de la Universidad de Buenos Aires en la historia del campo de las Relaciones Internacionales argentinas. Revista de Historia del Derecho, No. 52. Disponible en http://inhide.com.ar/portfolio/revista-de-historiadel-derecho-no-52-julio-diciembre-2016/ 
Dembicz, K. (2004). Los conceptos de la región y el desarrollo regional en el pensamiento socioeconómico de la CEPAL. Actas L. de V., 4, 69-76.

Di Tella, G. \& Zymelman, M. (1967). Las etapas del desarrollo económico argentino. Buenos Aires: Editorial Universitaria de Buenos Aires.

Dosman, E. (2008). The life and time of RaulPrebisch (1901-1986). s.1.: McGillsQueen's University Press.

Dosse, F., 2007. La marcha de las ideas: historia de los intelectuales, historia intelectual. Valencia: PUV.

Frieden, J. \& Lake, D. (2000). International Political Economy. Perspectives on global power and wealth. Londres/Nueva York: Routledge.

Gunder Frank, A. (1970). Latin America: Underdevelopment and revolution. Monthly Review Press.

Guzzini, S. (1998). Realism in International Relations and International Political Economy. The continuing story of the death foretold. Londres/Nueva York: Routledge.

Hirschman, A. (1968). The political economy of import-substituting industrialization in Latin America. Quarterly Journal of Economics, 82(1), 1-32.

Lipset, S. M. (1959). Some social requisites of democracy. American Political Science Review, 53(1), 69-105.

Lowenthal, A. (1983). Research in Latin America and the Caribbean on International Relations and foreign policy: Some impressions. Latin American Research Review, 18(1), 154-174.

Mace, G. (1988). Regional integration in Latin America: A long and winding road. International Journal, 43(3), 404-427.

Malamud, A. (2010). Latin American regionalism and EU studies. European Integration, 32(6), 637-657.

Morgenthau, H. (1948). Politics among nations. The struggle for power and peace. s.l.: McGraw-Hill Humanities/Social Sciences/Languages.

O’Donnell, G. (1973). Modernization and bureaucratic-authoritarianism. Los Ángeles: University of California Press.

Perrotta, D. (2014). La integración regional en América del Sur como objeto de estudio: ¿En pos de la construcción de un campo? Paper presentado en la Conferencia FLACSO-ISA, Buenos Aires, 23-25 de julio.

Plotkin, M. (2006). Economía y Psicología: la conformación de dos campos disciplinarios. En: M. Plotkin (Ed.), La privatización de la educación superior y las ciencias sociales en Argentina. Un estudio de las carreras de Psicología y Economía (pp. 25-42). Buenos Aires: CLACSO. 
Plotkin, M. (2010). La recepción y circulación de ideas económicas en la Argentina y las crisis de la segunda mitad del siglo XX. Buenos Aires: Primer Simposio Internacional Interdisciplinario "Aduanas del conocimiento": La traducción y la constitución de las disciplinas entre el centenario y el bicentenario.

Prebisch, R. (1950). The economic development of Latin America and its principal problems. Santiago de Chile: CEPAL.

Rostow, W. W. (1961). Las etapas del crecimiento económico. México: Fondo de Cultura Económica.

Scott, J. (2009). De-coding new regionalism: Shifting socio-political contexts in Central Europe and Latin America. Farnham: Ashgate Publishing.

Tickner, A. (2003). Hearing Latin American voices in international relations studies. International Studies Perspectives, 4.

Tussie, D. (2004). Of reason and purpose: Re-thinking the study of international relations in Latin America. Montreal: ISA.

Tussie, D. (2009). Latin America: Contrasting motivations for regional projects. Review of International Studies, 35, 169-188.

Tussie, D. (2015). Relaciones Internacionales y Economía Política Internacional: notas para el debate. Relaciones Internacionales, 24(48), 155-175.

Tussie, D. \& Riggirozzi, P. (2015). A global conversation: Rethinking IPE in post hegemonic scenarios. Contexto Internacional, 37(3), 1041-1068. 